\section{TEP na gravidez}

A incidência de TEV, incluindo TVP e TEP, encontra-se aumentada em cerca de 5 vezes durante a gravidez e o puerpério, em comparação com mulheres não grávidas na mesma idade, representando 1:2.000 grávidas. ${ }^{(1,2)}$ Diferentemente do diagnóstico em pessoas não grávidas, poucos estudos foram realizados com esse objetivo especifico. A extrapolação dos métodos para as grávidas pode não ser a forma correta na escolha do teste diagnóstico, mas, em muitas situações, essa extrapolação é utilizada. $^{(3)}$

Os sinais e sintomas de TEP em grávidas são de menor valor que em pacientes não grávidas, pois muitas alterações fisiológicas e anatômicas durante a gravidez podem mimetizar sintomas de TEP. ${ }^{(4)}$ A estimativa da probabilidade clínica pré-teste é fundamental na investigação de TEV e se baseia na presença de sintomas e sinais, em fatores de risco para TEV e na possibilidade de um diagnóstico alternativo, geralmente classificando as pacientes em alto, intermediário ou baixo risco. Existem vários escores de predição clínica, mas que não foram validados para gestantes. A simples extrapolação desses dados pode gerar erros, pois a população de gestantes é mais jovem, há diferentes fatores de risco não incluídos (por ex., síndrome de hiperestimulação ovariana) e nítida predominância de TVP no membro inferior esquerdo, os quais não foram incluídos no modelo. Mesmo assim, a impressão clínica subjetiva é importante.(D)

0 algoritmo de investigação de TEV em gestantes apresenta várias incertezas. Apesar de nenhum grande estudo utilizando a ecografia venosa de compressão e/ou Doppler de membros inferiores ter sido realizado em grávidas com suspeita de TEP, não há razões biológicas que impeçam a confirmação diagnóstica quando os achados são compatíveis com TVP, devendo, portanto, por ser praticamente um teste diagnóstico não invasivo, ser o exame inicial na suspeita de TEP em grávidas.(D) ${ }^{(5,6)}$ Contudo, um exame negativo não pode ser definido como ausência de TVP, principalmente na grávida, situação em que a veia ilíaca é mais frequentemente comprometida, local em que esse exame tem baixa acurácia.

Em relação aos testes laboratoriais, a dosagem de dimeros $\mathrm{D}$ aumenta progressivamente durante a gravidez, podendo haver um elevado número de resultados falso-positivos. Valores normais de dímeros $\mathrm{D}$ têm o mesmo valor para excluir TEP em mulheres gestantes do que em não gestantes para suspeitas de baixa probabilidade. ${ }^{(7.8)}$

0 elevado percentual de estudos cintilográficos pulmonares normais e de baixa probabilidade confirmam que muitos dos sinais e sintomas compatíveis com TEP em grávidas provavelmente são causados pela mimetização de alterações fisiológicas e anatômicas que ocorre durante a gravidez. Resultados de cintilografia normal são seguros em afastar o diagnóstico, enquanto resultados anormais têm alta probabilidade em afirmar o diagnóstico. A maioria das gestantes são mulheres jovens e com baixa incidência de doença cardiopulmonar prévia, o que diminui o percentual de cintilografias pulmonares não diagnósticas (cerca de 25\%). ${ }^{(9)}$

A angio-TC, no diagnóstico de TEP, tem papel fundamental em pacientes não grávidas. Não existe um estudo especifico e definitivo em grávidas sobre esse tópico. Contudo, um inquérito realizado em serviços de radiologia nos EUA, ${ }^{(10)}$ demonstrou que $75 \%$ desses serviços utilizam a angio-TC e 53\% o utilizam como o exame inicial. $\mathrm{Na}$ avaliação de exposição e segurança fetal, dois estudos demonstraram a baixa irradiação com esse exame. Portanto, visto sua alta acurácia e por não apresentar risco maior para o feto, pode ser utilizado em grávidas com suspeita de TEP. ${ }^{(11,12)}$ Estudos retrospectivos sugerem que a angio-TC pode ser mais útil que a cintilografia para o diagnóstico de TEP em gestantes. ${ }^{(13)}$ A ressonância nuclear magnética não apresenta indicação, até o momento, no diagnóstico de grávidas com suspeita de TEP. A arteriografia pulmonar é raramente utilizada na gravidez. ${ }^{(14)}$

$\mathrm{Na}$ ausência de um diagnóstico objetivo de TVP, a cintilografia pulmonar perfusional ou a angio-TC de tórax deve ser realizada para confirmar ou excluir a presença de TEP.(B)

Os prejuízos da falta de diagnóstico, associado aos riscos do uso inapropriado de anticoagulação durante a gravidez, são superiores aos riscos da radiação empregada para um diagnóstico preciso. ${ }^{(15)}$ Quando doses menores que 50 mSv são utilizadas, os riscos para o feto são muito baixos. A irradiação utilizada para o diagnóstico de TEP é possível com uma exposição menor que 0,5 mSv. 0 Quadro 18 apresenta a 
Quadro 18 - Dose de irradiação no feto em exames de imagem.

\begin{tabular}{|lc|}
\hline \multicolumn{1}{|c|}{ Teste diagnóstico } & Irradiação, $\mathrm{mSv}$ \\
\hline Radiografia de tórax (com proteção abdominal) & $<0,5$ \\
Arteriografia pulmonar via femoral & $2,21-3,74$ \\
Arteriografia pulmonar via braquial & $<0,5$ \\
Cintilografia de perfusão 99mTc & $0,2-0,6$ \\
Cintilografia de ventilação 99mTc & $0,1-0,3$ \\
Cintilografia de ventilação $81 \mathrm{mKr}$ & 0,0001 \\
Angio-TC pulmonar & $0,003-0,13$ \\
\hline
\end{tabular}

dose de irradiação utilizada em exames diagnósticos na suspeita de TEP.

0 tratamento de TEP em gestantes segue os mesmos princípios gerais da terapêutica em pacientes não grávidas. Os cumarínicos ultrapassam a barreira placentária e não devem ser usados, sobretudo no primeiro trimestre da gravidez, pelo risco de teratogenicidade. ${ }^{(16)}$ As heparinas não ultrapassam essa barreira e são seguras para a mãe, para o feto e para o recémnascido, não causando teratogenicidade..$^{(17-19)}$ A HBPM tem vantagens em relação à HNF por causar menos trombocitopenia, menos osteoporose e ter maior facilidade posológica. ${ }^{(20,21)}$

A dose das heparinas deve ser a habitual, corrigida proporcionalmente ao ganho que ocorre durante a gravidez. ${ }^{(22)}$ Se disponível, a monitorização do fator anti-Xa deve ser periodicamente realizada, com alvo terapêutico de 0,35-0,70 $\mathrm{Ul} / \mathrm{mL}$, determinada $4 \mathrm{~h}$ após a dose s.c. As heparinas s.c. devem ser suspensas pelo menos $24 \mathrm{~h}$ antes do parto induzido ou cesariana. Devem ser reiniciadas quando a hemostasia permitir, em geral 12-24 h após o nascimento. 0 cumarínico deverá ser iniciado junto com a heparina no pós-parto imediato. 0 tratamento deve ser prolongado no mínimo por 6 semanas após o parto, período de maior incidência de TEV (com um tempo total mínimo de 6 meses). (A) ${ }^{(23-27)}$ Convém ressaltar que os cumarínicos e as heparinas podem ser utilizados durante a amamentação sem riscos para o recém-nascido.

Para realizar a profilaxia de TEP em mulheres grávidas, é necessário conhecer aquelas pacientes que apresentam um risco aumentado, como episódio anterior ou trombofilia confirmada por exame laboratorial. Para a avaliação da intensidade do risco, é conveniente classificar a situação da grávida em: 1) episódio anterior único por fator de risco passageiro; 2) episódio anterior único por causa idiopática; 3) episódio anterior único de TEP associado à trombofilia confirmada por laboratório; 4) dois ou mais episódios de TEP; e 5) trombofilia sem episódio de TEP anterior. ${ }^{(27)}$ Essas categorias são amplas, e o risco necessita ser individualizado.(D)

Mulheres com um episódio prévio de TEV têm uma possibilidade aumentada de TEV em uma próxima gravidez. A taxa exata de recorrência não está bem estabelecida. ${ }^{(28,29)}$ Em um estudo prospectivo, 125 grávidas com episódio anterior único de TEV foram acompanhadas, sem uso de heparina, durante uma nova gravidez. ${ }^{(30)}$ A pesquisa de trombofilia foi realizada em 95 das grávidas; a taxa total de recorrência foi de 2,4\% (3/125). Em 44 grávidas sem trombofilia, nas quais o fator de risco do episódio anterior foi transitório, não houve recorrência. Entre as pacientes com trombofilia ou com episódio anterior idiopático, $3(5,9 \%)$ apresentaram recorrência. Na síndrome antifosfolipídeo, a incidência de TEV também se encontra aumentada. ${ }^{(31)}$

Um estudo retrospectivo recente envolvendo um grande número de grávidas com episódio anterior de TEV mostrou uma recorrência, durante a gravidez e puerpério, de $6,2 \%$, naquelas que não receberam profilaxia, chamando a atenção de que a recorrência ocorreu principalmente no pós-parto e que nenhum evento ocorreu nas pacientes que receberam a profilaxia. ${ }^{(32)}$ A HBPM é eficaz e segura na profilaxia de TEV em grávidas, e a HNF também se mostrou eficaz. ${ }^{(19,33-38)}$

As gestantes podem receber profilaxia ativa (com HNF ou HBPM) ou permanecer em vigilância clínica. Em uma avaliação de custo-efetividade, utilizando-se a HBPM para a profilaxia, foi demonstrado que, nas pacientes com episódio anterior único de TEV e baixo risco (causa transitória e sem trombofilia), a observação clínica 
Quadro 19 - Contextos que envolvem profilaxia para TEV na gestação. ${ }^{a}$

Podem dispensar a profilaxia antes do parto:

- História de TEV isolada diretamente relacionada a evento trombogênico transitório (trauma ortopédico, complicação cirúrgica) sem trombofilia identificada

- Carreadores assintomáticos de trombofilias (exceto deficiência de antitrombina, homozigose para fator V Leiden ou mutação do gene da protrombina), sem história pessoal nem familiar de trombose, pela marcada variação na penetração das trombofilias

Profilaxia para TEV com heparina em baixas doses antes do parto:

- Carreadores de outras trombofilias, sem história pessoal de TEV, mas forte história familiar de TEV

- História pessoal de TEV, mas sem trombofilia identificada

- História de trombose relacionada a estados estrogênicos (uso de anticoncepcional ou gestação prévia)

- História de trombose e deficiência de proteína C ou de proteína S

- Presença de síndrome pós-trombótica significativa

Pacientes que necessitam profilaxia para TEV com dose ajustada de heparina:

- Deficiência de antitrombina, com ou sem história de TEV

- Síndrome antifosfolipídeo. Pacientes sem história de TEV e sem outros fatores de risco podem ser candidatos a profilaxia com baixa dose

- Homozigose para mutação do fator V Leiden ou mutação G20210A do gene da protrombina

- Heterozigose para ambos as mutações (fator V Leiden e mutação G20210A do gene da protrombina)

- Pacientes em uso crônico de cumarínicos por TEV recorrente, ameaçadora da vida (por ex., cerebral), ou trombose recente

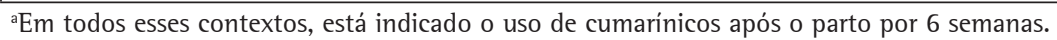

apresentou melhor relação de custo-efetividade que a profilaxia, diferentemente das pacientes com alto risco. 0 uso alternativo de HNF (5.000 U1 duas vezes ao dia) pode ser realizado, mas pode ser insuficiente para gestantes de alto risco. ${ }^{(27)} \mathrm{A}$ HBPM é, no momento, a medicação de escolha para a profilaxia e o tratamento de TEP.(B) ${ }^{(27,39)}$ Doses diárias baixas de HBPM para a profilaxia de TEV na gravidez são sugeridas (dalteparina, $5.000 \mathrm{Ul}$; enoxaparina, $40 \mathrm{mg} / \mathrm{dia}$; ou nadroparina, $2.850 \mathrm{UI}$ ). Doses profiláticas ajustadas de HBPM devem manter os níveis de antifator Xa 3-4 h após a injeção de 0,08-1,00 U1/mL. ${ }^{(27,40)}$

Em grávidas sem episódios prévios de TEV, mas com diagnóstico de trombofilia, a amplitude do risco e a incidência de um evento de TEV durante a gravidez dependem da alteração genética. A maior incidência ocorre em pacientes com deficiência de antitrombina, associação heterozigótica da protrombina G20210A, fator $\checkmark$ Leiden e em homozigóticos para uma dessas duas últimas alterações. ${ }^{(41-46)}$ Cerca de metade dos casos de TEV durante a gravidez está relacionada a essas alterações genéticas $(\mathrm{OR}=51,8$; 1C95\%: 38,0-69,2). ${ }^{(47)}$

Os principais cenários que ocorrem na gestação com a respectiva profilaxia estão relatados no Quadro 19.

A experiência com filtros de veia cava em mulheres grávidas está restrita a pequenas séries e a relatos de casos. Não há estudos que demonstrem sua eficácia e segurança por longos períodos, devendo ser utilizados em casos de exceção. ${ }^{(48)}$

\section{Referências}

1. Prevention of venous thrombosis and pulmonary embolism. NIH Consensus Development. JAMA. 1986;256(6):744-9.

2. Gherman RB, Goodwin TM, Leung B, Byrne JD, Hethumumi R, Montoro M. Incidence, clinical characteristics, and timing of objectively diagnosed venous thromboembolism during pregnancy. Obstet Gynecol. 1999;94(5 Pt 1):730-4. 
3. Nijkeuter M, Ginsberg JS, Huisman MV. Diagnosis of deep vein thrombosis and pulmonary embolism in pregnancy: a systematic review. J Thromb Haemost. 2006;4(3):496-500.

4. Stone SE, Morris TA. Pulmonary embolism during and after pregnancy. Crit Care Med. 2005;33(10 Suppl):S294-300.

5. Bates SM, Ginsberg JS. How we manage venous thromboembolism during pregnancy. Blood. 2002;100(10):3470-8.

6. Macklon NS, Greer IA, Bowman AW. An ultrasound study of gestational and postural changes in the deep venous system of the leg in pregnancy. Br J Obstet Gynaecol. 1997;104(2):191-7.

7. Francalanci 1, Comeglio P, Liotta AA, Cellai AP, Fedi $\mathrm{S}$, Parretti E, et al. D-dimer concentrations during normal pregnancy, as measured by ELISA. Thromb Res. 1995;78(5):399-405.

8. Proietti AB, Johnson MJ, Proietti FA, Repke JT, Bell WR. Assessment of fibrin(ogen) degradation products in preeclampsia using immunoblot, enzyme-linked immunosorbent assay, and latex-based agglutination. Obstet Gynecol. 1991;77(5):696-700.

9. Chan WS, Ray JG, Murray S, Coady GE, Coates G, Ginsberg JS. Suspected pulmonary embolism in pregnancy: clinical presentation, results of lung scanning, and subsequent maternal and pediatric outcomes. Arch Intern Med. 2002;162(10):1170-5.

10. Schuster ME, Fishman JE, Copeland JF, Hatabu H, Boiselle PM. Pulmonary embolism in pregnant patients: a survey of practices and policies for CT pulmonary angiography. AJR Am J Roentgenol. 2003;181(6):1495-8.

11. Winer-Muram HT, Boone JM, Brown HL, Jennings SG, Mabie WC, Lombardo GT. Pulmonary embolism in pregnant patients: fetal radiation dose with helical CT. Radiology. 2002;224(2):487-92.

12. Nijkeuter M, Geleijns J, De Roos A, Meinders AE, Huisman MV. Diagnosing pulmonary embolism in pregnancy: rationalizing fetal radiation exposure in radiological procedures. J Thromb Haemost. 2004;2(10):1857-8.

13. Cahill AG, Stout MJ, Macones GA, Bhalla S. Diagnosing pulmonary embolism in pregnancy using computedtomographic angiography or ventilation-perfusion. Obstet Gynecol. 2009;114(1):124-9.

14. Barbour LA; ACOG Committee on Practice Bulletins-Obstetrics. ACOG practice bulletin. Thromboembolism in pregnancy. Int J Gynaecol Obstet. 2001;75(2):203-12.

15. Ginsberg JS, Hirsh J, Rainbow AJ, Coates G. Risks to the fetus of radiologic procedures used in the diagnosis of maternal venous thromboembolic disease. Thromb Haemost. 1989;61(2):189-96.

16. Hall JG, Pauli RM, Wilson KM. Maternal and fetal sequelae of anticoagulation during pregnancy. Am J Med. 1980;68(1):122-40.

17. Forestier F, Daffos F, Rainaut M, Toulemonde F. Low molecular weight heparin (CY 216) does not cross the placenta during the third trimester of pregnancy. Thromb Haemost. 1987;57(2):234.

18. Sanson BJ, Lensing AW, Prins MH, Ginsberg JS, Barkagan $\mathrm{ZS}$, Lavenne-Pardonge E, et al. Safety of low-molecularweight heparin in pregnancy: a systematic review. Thromb Haemost. 1999;81(5):668-72.

19. Lepercq J, Conard J, Borel-Derlon A, Darmon JY, Boudignat O, Francoual C, et al. Venous thromboembolism during pregnancy: a retrospective study of enoxaparin safety in 624 pregnancies. BJOG. 2001;108(11):1134-40.

20. Warkentin TE, Levine MN, Hirsh J, Horsewood P, Roberts RS, Gent M, et al. Heparin-induced thrombocytopenia in patients treated with low-molecular-weight heparin or unfractionated heparin. $\mathrm{N}$ Engl J Med. 1995;332(20):1330-5.

21. Pettilä V, Leinonen P, Markkola A, Hiilesmaa V, Kaaja R. Postpartum bone mineral density in women treated for thromboprophylaxis with unfractionated heparin or LMW heparin. Thromb Haemost. 2002;87(2):182-6.

22. Crowther MA, Spitzer K, Julian J, Ginsberg J, Johnston $\mathrm{M}$, Crowther R, et al. Pharmacokinetic profile of a low-molecular weight heparin (reviparin) in pregnant patients. A prospective cohort study. Thromb Res. 2000;98(2):133-8.

23. Gerhardt A, Scharf RE, Beckmann MW, Struve S, Bender HG, Pillny M, et al. Prothrombin and factor V mutations in women with a history of thrombosis during pregnancy and the puerperium. N Engl J Med. 2000;342(6):37480.

24. Middeldorp S, Libourel EJ, Hamulyák K, Van der Meer $\mathrm{J}$, Büller HR. The risk of pregnancy-related venous thromboembolism in women who are homozygous for factor V Leiden. Br J Haematol. 2001;113(2):553-5.

25. Martinelli 1, Bucciarelli P, Zighetti ML, Cafro A, Mannucci PM. Low risk of thrombosis in family members of patients with hyperhomocysteinaemia. $\mathrm{Br} \mathrm{J}$ Haematol. 2002;117(3):709-11.

26. Martinelli I, De Stefano V, Taioli E, Paciaroni K, Rossi E, Mannucci PM. Inherited thrombophilia and first venous thromboembolism during pregnancy and puerperium. Thromb Haemost. 2002;87(5):791-5.

27. Bates SM, Greer IA, Pabinger I, Sofaer S, Hirsh J; American College of Chest Physicians. Venous thromboembolism, thrombophilia, antithrombotic therapy, and pregnancy: American College of Chest Physicians Evidence-Based Clinical Practice Guidelines (8th Edition). Chest. 2008;133(6 Suppl):844S-886S.

28. Howell R, Fidler J, Letsky E, de Swiet M. The risks of antenatal subcutaneous heparin prophylaxis: a controlled trial. Br J Obstet Gynaecol. 1983;90(12):1124-8.

29. Tengborn L, Bergqvist D, Mätzsch T, Bergqvist A, Hedner $\mathrm{U}$. Recurrent thromboembolism in pregnancy and puerperium. Is there a need for thromboprophylaxis? Am J Obstet Gynecol. 1989;160(1):90-4.

30. Brill-Edwards P, Ginsberg JS, Gent M, Hirsh J, Burrows R, Kearon C, et al. Safety of withholding heparin in pregnant women with a history of venous thromboembolism. Recurrence of Clot in This Pregnancy Study Group. N Engl J Med. 2000;343(20):1439-44.

31. Turiel M, Sarzi-Puttini P, Peretti R, Rossi E, Atzeni F, Parsons W, et al. Thrombotic risk factors in primary antiphospholipid syndrome: a 5-year prospective study. Stroke. 2005;36(7):1490-4.

32. Pabinger 1, Grafenhofer H, Kaider A, Kyrle PA, Quehenberger P, Mannhalter C, et al. Risk of pregnancyassociated recurrent venous thromboembolism in women with a history of venous thrombosis. J Thromb Haemost. 2005;3(5):949-54.

33. Hunt BJ, Doughty HA, Majumdar G, Copplestone A, Kerslake S, Buchanan N, et al. Thromboprophylaxis with low molecular weight heparin (Fragmin) in high risk pregnancies. Thromb Haemost. 1997;77(1):39-43. 
34. Nelson-Piercy C, Letsky EA, de Swiet M. Low-molecularweight heparin for obstetric thromboprophylaxis: experience of sixty-nine pregnancies in sixty-one women at high risk. Am J Obstet Gynecol. 1997;176(5):1062-8.

35. Brennand JE, Walker ID, Greer IA. Anti-activated factor $X$ profiles in pregnant women receiving antenatal thromboprophylaxis with enoxaparin. Acta Haematol. 1999;101(1):53-5.

36. Pettilä V, Kaaja R, Leinonen P, Ekblad U, Kataja M, lkkala E. Thromboprophylaxis with low molecular weight heparin (dalteparin) in pregnancy. Thromb Res. 1999;96(4):275-82.

37. Ellison J, Walker ID, Greer IA. Antenatal use of enoxaparin for prevention and treatment of thromboembolism in pregnancy. BJOG. 2000;107(9):1116-21.

38. Dahlman TC. Osteoporotic fractures and the recurrence of thromboembolism during pregnancy and the puerperium in 184 women undergoing thromboprophylaxis with heparin. Am J Obstet Gynecol. 1993;168(4):1265-70.

39. Greer 1A. Venous thromboembolism and anticoagulant therapy in pregnancy. Gend Med. 2005;2 Suppl A:S10-7.

40. Bazzan M, Donvito V. Low-molecular-weight heparin during pregnancy. Thromb Res. 2001;101(1):V175-86.

41. McColl MD, Ramsay JE, Tait RC, Walker ID, McCall F, Conkie JA, et al. Risk factors for pregnancy associated venous thromboembolism. Thromb Haemost. 1997;78(4):1183-8.
42. Gerhardt A, Scharf RE, Beckmann MW, Struve S, Bender HG, Pillny M, et al. Prothrombin and factor V mutations in women with a history of thrombosis during pregnancy and the puerperium. N Engl J Med. 2000;342(6):37480.

43. Middeldorp S, Libourel EJ, Hamulyák K, Van der Meer J, Büller HR. The risk of pregnancy-related venous thromboembolism in women who are homozygous for factor V Leiden. Br J Haematol. 2001;113(2):553-5.

44. Martinelli 1, De Stefano V, Taioli E, Paciaroni K, Rossi E, Mannucci PM. Inherited thrombophilia and first venous thromboembolism during pregnancy and puerperium. Thromb Haemost. 2002;87(5):791-5.

45. Robertson $\mathrm{L}, \mathrm{Wu} \mathrm{O}$, Langhorne $\mathrm{P}$, Twaddle S, Clark $\mathrm{P}$, Lowe GD, et al. Thrombophilia in pregnancy: a systematic review. Br J Haematol. 2006;132(2):171-96.

46. Greer 1A. Thrombosis in pregnancy: maternal and fetal issues. Lancet. 1999;353(9160):1258-65.

47. James AH, Jamison MG, Brancazio LR, Myers ER. Venous thromboembolism during pregnancy and the postpartum period: incidence, risk factors, and mortality. Am J Obstet Gynecol. 2006;194(5):1311-5.

48. Narayan H, Cullimore J, Krarup K, Thurston H, Macvicar J, Bolia A. Experience with the cardial inferior vena cava filter as prophylaxis against pulmonary embolism in pregnant women with extensive deep venous thrombosis. Br J Obstet Gynaecol. 1992;99(8):637-40. 